\title{
Twenty-second Annual Saskatchewan Christmas Bird Count, 1963
}

\author{
Edited by Mary Houston, Saskatoon
}

This year only 23 Saskatchewan localities reported Christmas Bird Counts-the lowest number since 1957. Fifty species of birds were seen on count day-again the lowest number since 1957.

Two new species bring the total list of birds seen in 22 years of Christmas bird counts in Saskatchewan to 110. A Rufous-sided Towhee was seen at Mcose Jaw. From Lady Lake, Donald Buckle reported what he believes was a Gyrfalcona large, light greyish white bird, near the size of a Snowy or Great Horned Owl with a small hawk-like head, and a long tail lightly barred on its underside. He flushed this bird three times from a spruce grove bordering the Lilian River, and was within 50 feet of it when it was in flight.

There were no marked trends in species numbers except for Redpolls which were repcrted from 19 of the 23 localities compared to 13 of 26 localities in 1962. Other species most frequently seen were recorded in the following numbers of localities: House Sparrow, 22; Black-billed Magpie, 21; Black-capped Chickadee, 16; Snow Bunting, 13; Bohemian Waxwing, 12; Gray Partridge, 12; Downy Woodpecker, 11; Rock Dove, 11; Pine Grosbeak, 10; Great Horned Owl, 10; Sharp-tailed Grcuse, 9.

BANGOR, Sask. Dec. 27; 30 miles by car and around yard; a few degrees below zero; sunny. 6 species, 559 individuals. Boreal Owl, 1; Black-billed Magpie, 2; Black-capped Chickadee, 4; Hcuse Sparrow, 53; Common Redpoll, 50; Snow Bunting, 449. (Add: Sharp-tailed Grouse, 3, Dec. 24; Great Horned Owl, 1, Dec. 24; Downy Woodpecker, 1, Dec. 24; Pine Grosbeak, 15, Dec. 24).-Mrs.

\section{A. Thompson.}

BATTLEFORD, Sask. Dec. $31 ; 39$ miles by car and 6 miles on foct in $51 / 2$ hours; temp $39^{\circ}$; wind N.W. at 8 m.p.h.; 4 inches of snow. 16 species, 357 individuals. Ruffed Grouse, .5; Sharp-tailed Grouse, 91; Gray Partridge, 1; Rock Dove, 3; Great Horned Owl, 3; Hairy Woodpecker, 1; Blue
Jay, 1; Black-billed Magpie, 32; Black-capped Chickadee, 14; Bohemian Waxwing, 7; Northern Shrike, 1; Hcuse Sparrow, 53; Evening Grosbeak, 19; Pine Grosbeak, 9; Common Redpoll, 44; Snow Bunting, 73.Spencer G. Sealy.

BLADWORTH, Sask. Dec. 27; 4 hours afield by truck and on foot, then around the yard the rest of the day; temp. $-6^{\circ}$ to $2^{\circ}$; almost calm; variable clcudiness. 9 species, 174 individuals. Sharp-tailed Grcuse, 4; Gray Partridge, 23; Rock Dove, 3; Great Horned Owl, 1; Downy Woodpecker, 1; Black-billed Magpie, 11; Black-capped Chickadee, 1; House Sparrow, 60; Common Redpoll, 70. (Add: Golden Eagle, 1, Dec. 22; Hcrned Lark, 3, Dec. 22; Bchemian Waxwing, 3, Dec. 20, 23; Rusty Blackbird, 1, Dec. 22, 23; Evening Grosbeak, 1, Dec. 21, 30).-Lawrence Beckie.

BUFFALO POUND LAKE, Sask. Dec. $27 ; 14$ party hours, $13 \frac{1}{2}$ on foot and $1 / 2$ by car, and $201 / 2$ miles by foot and 18 by car; temp $10^{\circ}$ to $19^{\circ}$; wind N.W. 15 m.p.h.; clear; 3 inches of snow on the level; prairie, hills, wooded draws. 10 species, 222 individuals. Sharp-tailed Grouse, 25; Great Horned Owl, 1; Short-eared Owl, 2; Hairy Woodpecker, 1; Downy Woodpecker, 2; Black-billed Magpie, 19; Common Crow, 1; Pine Grosbeak, 5; Common Redpoll, 137; Snow Bunting, 29.-Mr. Carl Ellis, John Ellis, John Horton and Michael Rhodes (compilers).

DILKE, Sask. Dec. 22; 34 miles in 3 hours by car, 3 miles on foot and 3 hours abcut farmyard; temp $0^{\circ}$ to $10^{\circ}$; wind light rising to gusts of 35 m.p.h. by afternoon; snow cover 2 inches. 6 species, 400 individuals. Sharp-tailed Grouse, 19; Horned Lark, 1; Black-billed Magpie, 28; House Sparrow, 81; Rusty Blackbird, 1; Common Redpoll, 270. (Add: Gcshawk, 1, Dec. 23; Golden Eagle, 1, Dec. 25; Gray Partridge, 7, Dec. 31; Great Horned Owl, 1, Dec. 28; Snowy Owl, 1, Dec. 28, 30, 31; Black-capped Chickadee, 1-2, Dec. 20, 21, 23, 25, 31 ; Bohemian Waxwing 2, Dec. 27; 
Northern Shrike, 1, Dec. 23, 25, 27, 29).-Boswell Belcher (compiler), Margaret Belcher, Mr. and Mrs. S. R. Belcher.

ESTEVAN, Sask. Dec. 27; 6 miles by fcot and 110 miles by car in 8 hours; temp $5^{\circ}$, rising to $15^{\circ}$; wind calm, rising to N.W. 20 m.p.h. at noon; 4 inches of snow. 20 species, 909 individuals. Mallard, 130; Lesser Scaup, 1; Sharp-tailed Grouse, 15; Gray Partridge, 12; Rock Dove, 4; Great Horned Owl, 1; Short-eared Owl, 1; Downy Wocdpecker, 1; Horned Lark, 144; Black-billed Magpie, 5; Blackcapped Chickadee, 5 ; Bchemian Waxwing, 33; Starling, 11; House Sparrow, 300; Red-winged Blackbird, 10; Rusty Blackbird, 10; Pine Grosbeak, 17; Ccmmon Redpoll, 100; Tree Sparrow, 4; Snow Bunting, 105. (Add: Golden Eagle, 1, Dec. 23; Prairie Falcon, 1, Dec. 23).-Darrel Carlson, M. Ross Lein (compiler).

ETHELTON, Sask. Dec. 26; 15 miles by car and 3 miles on foot in 3 hours; temp. $0^{\circ}$; wind light; light snow falling; 12 inches of snow on ground. 6 species, 178 individuals. Gray Partridge, 7; Hairy Woodpecker, 1; Downy Wcodpecker, 1; Black-billed Magpie, 7; Black-capped Chickadee, 6; House Sparrow, 156. (Add: Snow Bunting, 300, Dec. 25.)-Genevieve Belliveau (compiler), Mrs. George Clarke.

FORT QU'APPELLE, Sask. Dec. 21; temp. $5^{\circ} ; 2$ inches of sncw. 14 species, 414 individuals. Mallard, 1; Roughlegged Hawk, 1; Ruffed Grouse, 1; Sharp-tailed Grouse, 3; Downy Woodpecker, 2; Blue .Jay, 1; Black-billed Magpie, 5; Black-capped Chickadee, 5; Bohemian Waxwing, 36; Northern Shrike, 1; House Sparrow, 41; Rusty Blackbird, 1; Pine Grosbeak, 1; Common Redpoll, 315. (Add: Goshawk, 1, Dec. 24, 29; Golden Eagle, 2, Dec. 24, 25; Gray Partridge, 11, Dec. 25; Rcck Dove, 11, Dec. 26; Great Horned Owl, 1, Dec. 26, 28; Snowy Owl, 1, Dec. 20; Hairy Wocdpecker; Whitebreasted Nuthatch, 1, Dec. 23; Redwinged Blackbird, 1, Dec. 23, and 4, Dec. 29; Slate-colored Junco, 1; Oregcn Junco, 1, Jan. 1; Snow Bunting, 35, Dec. 22)-Dr. and Mrs. G. D. Barnett, Alden Barnett, E. Manley Callin (compiler), Richard Carter, Mr. and Mrs. Errol Cochrane, Mr. and Mrs. William Gray, Richard Nevard, Horace Reed, S. Regan, Mr. and Mrs. Rumancik.
GRENFELL, Sask. Dec. 24; 1 hour and 1 mile on foot, and around farm all day; temp. $38^{\circ}$; calm; overcast; summerfallow bare, 3-4 inches snow in stubble; parkland. 7 species, 188 individuals. Black-billed Magpie, 5; Black-capped Chickadee, 2; Bohemian Waxwing, 19; Hcuse Sparrow, 100; Common Redpoll, 60; Slate-colored Junco, 2 (1 very rusty brown but with no distinct head marking); (Add: Sharp-tailed Grouse, 32, Dec. 29; Gray Partridge, 8, Dec. 28; Great Horned Owl, 1, Dec. 27; Hairy Woodpecker, 1, Dec. 29; Northern Shrike, 1, Dec. 28; Pine Grosbeak, 4, Dec. 28)-Mr. and Mrs. John Hubbard.

HA WARDEN, Sask. Dec. 24; 50 miles by car, and around the farmyard on foct; $6 \frac{1}{2}$ hours; temp. $35^{\circ} ; 3$ to 4 inches of snow; calm. 9 species, 461 individuals. Gray Partridge, 12; Rock Dove, 17; Snowy Owl, 1; Black-billed Magpie, 9; Black-capped Chickadee, 1; Bohemian Waxwing, 1; House Sparrow, 200; Ccmmon Redpoll, 20; Snow Bunting, 200. (Add: Sharptailed Grouse, 2, Dec. 27, and 7, Dec. 31; Great Horned Owl, 1, Dec. 29; Short-eared Owl, 1, Dec. 27.)-Harold Kvinge.

KINDERSLEY, Sask. Dec. 26; 18 miles by car, 2 miles on foot; temp. $5^{\circ}$; wind 5 m.p.h.; sunny. 7 species, 541 individuals. Gray Partridge, 100; Rock Dove, 2; Great Horned Owl, 1; Bchemian Waxwing, 46; Starling, 2; House Sparrow, 190; Snow Bunting, 200. (Add: Golden Eagle, 1, Dec. 28; Snowy Owl, 1, Dec. 28; Common Redpoll, 4, Dec. 31)-Glen A. Fox (compiler), Ron and Kim Lamont.

LADY LAKE, Sask. Jan. 1; 16 miles in 1 hour by car, 1 hour on foot; temp. $30^{\circ}$; clear and calm; 8 inches of snow. 9 species, 39 individuals. Gyrfalcon, 1 (see introduction-Ed.); Gray Jay, 2; Black-billed Magpie, 2; Common Raven, 1; Black-capped Chickadee, 5; Boreal Chickadee, 2; House Sparrow, 3; Pine Grosbeak, 3; Snow Bunting, 20. (Add: Great Horned Owl, 1, Dec. 23; Bohemian Waxwing, 3, Dec. 30)-Donald J. Buckle.

LLOYDMINSTER, Sask. Dec. $29 ; 40$ miles by car and on foot in 3 hours; temp. $21^{\circ}$; wind S.W. 4 m.p.h.; partly clcudy. 12 species, 166 individuals. Ruffed Grouse, 1; Great Horned Owl, 1; Blue Jay; 6; Black-billed Magpie, 13; Black-capped. . Chickadee, .12; Bohemian Waxwing, 11; Starling, 4; 
House Sparrow, 48; Evening Grosbeak, 7; Common Redpoll, 35; Red Crossbill, 4; Snow Bunting, 24.) -Russ Robertson.

MASEFIELD, Sask. Dec. 24; 35 miles by car and 3 miles on foot along the river; temp. $35^{\circ}$; calm; sunny, misting later; no snow on the level. 10 species, 383 individuals. Ring-necked Pheasant, 5; Gray Partridge, 4; Horned Lark, 10; Black-billed Magpie, 10; Black-capped Chickadee, 1; Bohemian Waxwing, 10; House Sparrow, 300; Rusty Blackbird, 12; Common Redpoll, 25; Tree Sparrow, 6. (Add: Golden Eagle, Dec. 23; Pigeon Hawk, Dec. 25; Snowy Owl, Dec. 25; Long-eared Owl, Dec. 21; Northern Shrike, Dec. 29; Snow Bunting, Dec. 21.)-J. David Chandler.

MOOSE JAW, Sask. Dec. 26; 12 party miles in 9 hours on foot, and 19 party miles in 12 party hours by car; temp. $10^{\circ}$ to $12^{\circ}$; wind gusting to 30 m.p.h.; cloudy, clearing later; 4 inches of snow; town, river valley, prairie. 13 species, 456 individuals. Ring-necked Pheasant, 18; Rock Dove, 16; Downy Woodpecker, 1; Black-billed Magpie, 22; Black-capped Chickadee, 8; Bohemian Waxwing, 12; Starling, 1; House Sparrow, 300; Red-winged Blackbird, 1; Common. Redpoll, 27; Rufous-sided Towhee, 1; Slatecolored Junco, 3; Snow Bunting, 46. (Add: Brewer's Blackbird, 1, Dec. 29; Pine Grosbeak, 9, Dec. 29,' and '4, Dec. 30; Oregon Junco, 1, Dec. 23.)J. Horton and M. Rhodes (compilers), John Ellis, Mrs. Vesta Humphreys, Mr. and Mrs. C. Knight, Mr. and Mrs. J. Nelson, Mrs. A. Rankin, Mrs. Dorothy Rhodes, Mrs. C. V. Stokins, Mrs. F. B. Taylor, Mrs. C. West.

REGINA, Sask. Dec. 28; party hours $1081 / 2$ ( 43 on foot, $651 / 2$ by car); party miles $3131 / 2 \quad(301 / 2$ on foot, 283 by car); temp. $-12^{\circ}$ to $6^{\circ}$; calm and clear. 29 species, 6823 individuals. Mute Swan, 11; Whistling Swan, 3; Canada Goose, 390; Mallard; 990; American. Widgeon, 7; Lesser Scaup, 2; Common Goldeneye, 2; Ruddy Duck, 3; Pigeon Hawk, 1; Ringnecked Pheasant, 7; Gray Partridge, 30; American Coot, 11; Rock Dove, 156; Great Horned Owl, 4; Snowy Owl, 5; Short-eared Owl, 7; Downy Wocdpecker, 7; Horned Lark, 2; Black-billed Magpie, 113; Blackcapped Chickadee, 29; Boreal Chick- adee, 3; Bohemian Waxwing, 153; Northern Shrike, 3; Starling, 8; House Sparrow, 2550; Rusty Blackbird, 1; Pine Grosbeak, 83; Common Redpoll, 462; Snow Bunting, 1780. (Add: Gadwall, 2, Dec. 24; Pintail, 1, Dec. 24; Blue-winged Teal, 1, Dec. 24; Canvasback, 1, Dec. 24; Prairie Falcon, 1, Dec. 30 ; Yellow-shafted Flicker, 1, Dec. 26; Hairy Woodpecker, 1, Dec. 29 and Jan. 1; White-breasted Nuthatch, 1, Jan. 1; Robin, 1, Dec. 26; Hoary Redpoll, 3, Jan. 1; Whitewinged Crossbill, 8, Dec. 29; Slatecolored Junco, 3, Dec. 19.)-Ron Austin, Mrs. A. J. Bailey, Margaret Belcher, Mr. and Mrs. Al Binnie, Frank Brazier, Betty Cruickshank, Elmer Fox. Reg Fox, Doug Gilroy, Sharon Haggerty, George and Marjorie Ledingham, Mr. and Mrs. Fortescue McKay, Sally Moss, Robert Nero, Carla Stein, Ruth and Herb Tempel, Margaret Tempel, Dot and Doug Wade (compilers), Holly Wallace, Vic Wilshire.

SASKATOON, Sask. Dec. 26; party hours $44(201 / 2$ on foot and $231 / 2$ by car); party miles 283 (34 on foot and 249 by car); temp. $4^{\circ}$ to $6^{\circ}$; wind N.W. 15 m.p.h.; partly cloudy; 4 inches of snow. 28 species, 6217 individuals. Mallard, 7; Common Goldeneye, 5; Pigeon Hawk, 2; Sharptailed Grouse, 106; Ring-necked Pheasant, 22; Gray Partridge, 127; Rock Dove, 276; Great Horned Owl, 5; Snowy Owl, 4; Short-eared Owl, 1; Yellow-shafted Flicker, 1; Hairy Wcodpecker, 1; Downy Woodpecker, 5; Blue Jay, 2; Black-billed Magpie, 137; Common Raven, 1; Black-capped Chickadee, 49; Red-breasted Nuthatch, 1; Bohemian Waxwing, 444; Northern Shrike, 6; Starling, 4; House Sparrow, 2467; Evening Grosbeak, 10; Pine Grosbeak, 61; Hoary Redpoll, 13; Common Redpoll, 1676; Red Crossbill, 8; Snow Bunting, 781. -Harold Belcher, W. J. Brucks, Malcolm Campbell, Pern Cordery, Bob Folker, Dr. and Mrs. John Gerrard, Chris, Peter and Jonathan Gerrard, Mr. and Mrs. J. B. Gollop, Michael Gollop, Mr. and Mrs. Jim Hogg, Dr. C. J. Houston, Dr. and Mrs. Stuart Houston, Stan Houston, Murray Martin, Jean McKenzie, Greg Michalenko, Mrs. Jane Miller, Jim Miller, Bob Mills, John Shadick, Stanley Shadick, Russ Shemko, Jim Slimmon, Alvena Schnell, Joe Turnquist, Jim Wedgwood. 
SPIRIT LAKE, Sask. Dec. 29; 4 hours and 4 miles on foot, and all day abcut the yard; snow depth $101 / 2$ inches. 11 species, 123 individuals. Ruffed Grouse, 1; Hairy Woodpecker, 6; Downy Woodpecker, 9; Blue Jay, 1; Black-billed Magpie, 2; Blackcapped Chickadee, 55; White-breasted Nuthatch, 4; House Sparrow, 20; Evening Grosbeak, 5; Pine Grosbeak, 2; Common Redpoll, 18. (Add: Sharptailed Grcuse, 8 on Dec. 31,22 on Dec. 30; Rock Dove, 1, Jan. 1; Great Horned Owl, 1, Dec. 23, 27, 28, 30; Snowy Owl, 1, Dec. 30; Bohemian Waxwing, 8, Dec. 22; Snow Bunting, 10, Dec. 27, 31.)-W. Anaka, J. Gunn.

SWIFT CURRENT, Sask. Dec. $30 ; 20$ miles by car, 4 miles on foot in 5 hours; temp. $19^{\circ}$ to $43^{\circ}$; wind W.S.W. at 22-30 m.p.h.; partly clcudy; light covering of snow in patches. 10 species, 926 individuals. Ring-necked Pheasant, 1; Gray Partridge, 12; Rock Dove, 12; Great Horned Owl, 1; Downy Woodpecker, 1; Black-billed Magpie, 5; Black-capped Chickadee, 5; Bohemian Waxwing, 13; House Sparrow, 800; Common Redpoll, 76. (Add: Rcbin, 1, Dec. 24; Pine Grosbeak, 4, Dec. 29; Slate-colored Junco, 1, Dec. 29.)-Bert Hare, Marian Hare, Vince Honig, Jean McDaid (compiler)

TULLIS, Sask. Dec. 27; 25 miles by car and 5 on fcot in $31 / 2$ hours; temp. $4^{\circ}$; no wind; clear, clouding afternoon; no snow. 6 species, 160 individuals. Sharp-tailed Grouse, 5; Gray Partridge, 30; Snowy Owl, 1; Blackbilled Magpie, 4; House Sparrow, 25; Ccmmon Redpoll, 95.-Bob Boon, Chris Boon, Mrs. E. Boon (compiler).

WOLSELEY, Sask. Dec. $31 ; 4$ miles on horseback, 20 miles by truck; 4 inches snow. 12 species, 430 individuals. Rock Dove, 4; Great Horned Owl, 1; Downy Wocdpecker, 1; Blackbilled Magpie, 8; Black-capped Chickadee, 3; Starling, 3; House Sparrow, $300+$; Red-winged Blackbird, 3; Brewer's Blackbird, 23; Pine Grosbeak, 2; Common Redpoll, 80; Hoary Redpoll, 2. (Add: Ruffed Grouse, 1, Dec. 30; Sharp-tailed Grcuse, 18, Dec. 20; Gray Partridge, 7, Dec. 23.)Donald Hayward.

WOODROW, Sask. Dec. 20; 49 miles by car; temp. $15^{\circ}$; calm; bright; 5 inches of snow; open country near Wood River and Pinto Creek. 11 species, 753 individuals. Ring-necked
Pheasant, 42; Sharp-tailed Grouse, 1; Gray Partridge, 49; Rcck Dove, 4; Snowy Owl, 1; Short-eared Owl, 1; Horned Lark, 150; Black-billed Magpie 2; Starling, 3; House Sparrow, 300; Snow Bunting, 200.-C. H. Shulver.

YELLOW CREEK, Sask. Jan. 1; 11/2 miles for 2 hours through farmyards and light brush; temp. $30^{\circ}$ to $35^{\circ}$; light wind; partly cloudy; 8 inches of sncw. 6 species, 102 individuals. Pil€ated Woodpecker, 1; Black-capped Chickadee, 7; House Sparrow, 52; Pine Grosbeak, 1; Commcn Redpoll, 12; Snow Bunting, 29. (Add: Hairy Wcodpecker, 2, Dec. 24; Downy Woodpecker, 3, Dec. 24; Black-billed Magpie, 2, Dec. 24.)-Bohdan and Irene Pylypec.

COLD LAKE, Alta. Dec. 28; 27 miles by car and 1 mile on foot in $2 \frac{1}{2}$ hours; temp. $2^{\circ}$; wind W.S.W. at 7 to 10 in.p.h.; cvercast with light snow; 4 inches of snow on the ground. 6 species, 38 individuals. Blue Jay, 2; Black-billed Magpie, 9; Common Raven, 3; Black-capped Chickadee, 6; Pine Grosbeak, 9; Common Redpoll, 9.-Frank Roy, S. West.

WETASKIWIN, Alta. Dec. 25; daylight hours abcut the 145-acre farm; temp. $24^{\circ}$ in a.m., drcpping to $5^{\circ}$ by evening; overcast, with morning fog; wind E. at 6 m.p.h., shifting to N.W. at 15 m.p.h. 9 species, 120 individuals. Ruffed Grouse, 1; Gray Partridge, 6; Downy Woodpecker, 1; Blue Jay, 2; Black-billed Magpie, 4; Black-capped Chickadee, 3; Bohemian Waxwing, 1; House Sparrow, 100; Pine Grosbeak, 2. (Add: Goshawk, 1, Dec. 31; Great Horned Owl, 1, Dec. 28; Northern Shrike, 1, Dec. 26; Evening Grosbeak, 1, Dec. 26.)-Fred H. Pegg:

FORT SIMITH, N.W.T. Dec. $29 ; 37$ miles by truck and 3 miles on sncwshces for 6 hours; temp. $-5^{\circ}$; wind S.S.W. at 10 m.p.h.; snow 2 feet deep in bush and 1 foot on lakes; mixed forest of jackpine, white and black spruce, birch and willow thickets and white pcplar stands; a number of small lakes and sedge marshes. 5 species, 56 individuals. Ruffed Grouse, 3; Willow Ptarmigan, 29; Gray Jay, 4; Common Raven, 12; Hoary Redpoll, 8. (Add: Boreal Chickadee, 1, Dec. 24; Pine Grosbeak, 22, Dec. 24.) Elsie and Ernie Kuyt. 